\title{
Assessment of the scattering by sub-micron particles in inland waters
}

\author{
M. L. Laanen \\ laanen@waterinsight.nl
}

\section{S. W. M. Peters}

\section{A. G. Dekker}

\section{H. J. van der Woerd}

Institute for Environmental Sciences, Vrije Universiteit Amsterdam, De Boelelaan 1087, 1081 HV Amsterdam, The Netherlands

Current address: Water Insight BV, Marijkeweg 22, 6709 PG Wageningen, The Netherlands

Institute for Environmental Sciences, Vrije Universiteit Amsterdam, De Boelelaan 1087, 1081 HV Amsterdam, The Netherlands

CSIRO Land and Water, Clunies Ross Street, Black Mountain ACT 2601, Australia

Institute for Environmental Sciences, Vrije Universiteit Amsterdam, De Boelelaan 1087, 1081 HV Amsterdam, The Netherlands

Current protocols prescribe $0.2 \mu \mathrm{m}$ filters for absorption measurements of Coloured Dissolved Organic Matter $\left(\mathrm{a}_{(\mathrm{CDOM})}\right)$. However, a large historical dataset of $\mathrm{a}_{(C D O M)}$ was obtained over $0.7 \mu \mathrm{m}$ filters. Since small particles may pass $0.7 \mu \mathrm{m}$ filters, $\mathrm{a}_{(C D O M)}$ was probably overestimated because of residual scattering. Furthermore, current protocols require suspended matter to be filtered over $0.7 \mu \mathrm{m}$. So the $0.2 \mu \mathrm{m}-0.7 \mu \mathrm{m}$ fraction is missing from the particles and attributed to enhanced $\mathrm{a}_{(C D O M)}$, potentially leading to poor optical closure. In this paper natural water samples from a eutrophic lake were analysed with both filter pore sizes and two different absorption determination methods. Besides a standard bench spectrometer with a cuvette a calibrated Point-Source integrating-Cavity Absorption Meter (PSICAM) was used to gauge the absorption measurements. After filtration over $0.7 \mu \mathrm{m}$ we found a systematic $8 \%$ overestimation of $\mathrm{a}_{(C D O M)}$ measured in the cuvette caused by residual scattering. By filtering over $0.2 \mu \mathrm{m}$ the $\mathrm{a}_{(C D O M)}$ cuvette values are $6 \%$ underestimated because also a significant fraction of absorption was removed from the sample. As a consequence we recommend testing for coloured residues on the $0.2 \mu \mathrm{m}$ filter after prime filtration with a glass-fibre (GF/F) filter. In case the $0.2 \mu \mathrm{m}$ filter shows colouration, the method presented in this paper is a fast and simple check to correct the CDOM measurements. [DOI: http://dx.doi.org/10.2971/jeos.2011.11046]

Keywords: Gilvin, CDOM, filtration protocols, scattering, absorption, lakes, PSICAM

\section{INTRODUCTION}

The colour of natural surface water is determined by the spectral characteristics and the concentrations of dissolved and suspended coloured compounds. The establishment of quantitative relationships between the water colour and the concentrations of these compounds is essential for accurate derivation of the concentration of optical active constituents from handheld and satellite spectral observations. Reflection spectra are due to the light scattering of particles and water molecules that reflect upward part of the solar irradiance. The spectral subsurface irradiance reflectance $R(0, \lambda)$ is a function of wavelength $(\lambda)$ and is related to the total backscatter coefficient $b_{b}(\lambda)$ and absorption coefficient $\mathrm{a}(\lambda)$ by [1]

$$
R(0, \lambda)=f \frac{b_{b}(\lambda)}{a(\lambda)+b_{b}(\lambda)}
$$

Here $\mathrm{f}$ is a scaling constant that depends on illumination conditions and the scattering properties of the water. The total backscattering is the sum of backscatter by water (w), phytoplankton (PH) and non-algal particles (NAP):

$$
b_{b}=b_{b(w)}+b_{b(P H)}+b_{b(N A P)}
$$

The total absorption is given by

$$
a=a_{(w)}+a_{(P H)}+a_{(N A P)}+a_{(C D O M)} .
$$

Here $a_{(C D O M)}$ is the absorption by the dissolved humic substances in the water (Gilvin or Coloured Dissolved Organic Matter). This three-component model is applied in most optical modelling studies and is minimally required to successfully simulate natural waters [2].

The absorption and scattering properties in equations Eq. (2) and Eq. (3) need to be determined accurately by laboratory reference measurements. By definition, the dissolved fraction should display absorption but no scattering. Currently, this fraction is defined in practise as the fraction that passes a $0.2 \mu \mathrm{m}$ filter [3]. In case of the filtration over $0.2 \mu \mathrm{m}(200 \mathrm{~nm})$ the particle size will always be less than half a wavelength in the visual region of interest $(400-750 \mathrm{~nm})$ and the resulting Rayleigh scattering is relatively small and can be ignored $[4,5]$. However, in literature other threshold values can be found (e.g. $0.45 \mu \mathrm{m}$, see [6]) and in operational water remote sensing studies the dissolved matter is measured after filtration over a GF/F glass-fibre filter (with a nominal pore size of $0.7 \mu \mathrm{m})$.

The filters that are used for $a_{(C D O M)}$ determination can not 
only vary in pore size but also in filter material. Two main types of filters are generally used: glass-fibre and celluloseacetate (or membrane) filters. Glass-fibre filters are relatively cheap, but are only available with relatively large pore sizes. The given pore size of a glass-fibre filter is commonly the nominal pore size. It is inherent to the material used that the pore sizes of a glass-fibre filter are not uniform; the distance between strings can vary between smaller than the nominal pore size up to the nominal given size. For instance, the Whatman GF/F filters used for this research have a nominal pore size of $0.7 \mu \mathrm{m}$, while the effective pore size is closer to about $0.45 \mu \mathrm{m}$ (http://www.whatman.com). Glass-fibre filters with smaller nominal pore sizes than $0.7 \mu \mathrm{m}$ are not available.

Until recently, in water remote sensing the particulate and dissolved matter was separated by filtration over a $0.7 \mu \mathrm{m}$ glassfibre filter [7]. The disadvantage of this methodology is that sub-micron particles, whose behaviour is more similar to the particulate matter (such as detrital matter, clay and picoplankton), still pass through to the filtrate. As was demonstrated by Simis et al [8] these particles cause extra scattering of light, for example in a cuvette beam attenuation measurement. In a cuvette of length $L[\mathrm{~m}]$ the beam attenuation $C(\lambda)\left[\mathrm{m}^{-1}\right]$ of the incoming light intensity $\mathrm{I}_{0}(\lambda)$ is derived from the outgoing light intensity $\mathrm{I}(\lambda)$ by

$$
C=L^{-1} \ln \left(\frac{I_{0}}{I}\right)
$$

The attenuation $C$ is composed of the losses at the cuvette air/quartz/water interfaces $(\Delta)$, attenuation by pure water and the absorption (a) and scattering (b) of the coloured compounds in the water.

$$
c=a_{(w)}+b_{(w)}+a+b+\Delta
$$

In a calibration measurement the sum term $\left(a_{(w)}+b_{(w)}+\Delta\right)$ is derived from a cuvette filled with Milli-Q water. The extra attenuation measured in the filtrate is regarded as the CDOM absorption of the sample. However, if a portion of this attenuation is caused by the scattering of photons $(b \neq 0)$ out of the light path, this will be wrongly attributed to the CDOM absorption measure.

Even though the CDOM protocol was changed to filtration over $0.2 \mu \mathrm{m}$, the measurement protocols for the concentrations and absorptions (filter-pad method [9]) of phytoplankton and NAP still call for a filtration over a $0.7 \mu \mathrm{m}$ filter. A filtration over smaller pore size filters is often not feasible for particulate matter in inland waters with high concentrations of optically active compounds. Therefore the weight of the particulate fraction between 0.7 to $0.2 \mu \mathrm{m}$ is missing and the pigment concentration in the pico-plankton fraction is probably missing. In addition the absorption by the NAP and phytoplankton fraction between 0.7 and $0.2 \mu \mathrm{m}$ is also not measured. This will affect optical closure between measured and simulated spectra and will also affect the concentration retrievals of CDOM, NAP and probably also of phytoplankton.

In this paper we assess the magnitude of the scattering of submicron particles by comparing the attenuation properties of the filtrate after passing a $0.7 \mu \mathrm{m} \mathrm{GF} / \mathrm{F}$ glass fibre filter to the optical attenuation after filtration over an $0.2 \mu \mathrm{m}$ celluloseacetate filter. The attenuation measurements are made with a cuvette and with the integrating-cavity absorption meter [10], in this case the Point-Source Integrating-Cavity Absorption Meter (PSICAM). Since the light field inside an integrating cavity is already totally diffuse, the absorption measured by the PSICAM is assumed to be free of scattering [11]. Another advantage of the PSICAM over cuvette measurements is that the average path length (L) of photons that can be achieved in the cavity is much longer because of multiple reflections at the cavity wall (in our case around $1 \mathrm{~m}$ ). This allows the PSICAM to measure with high accuracy and at very low absorption. A number of case studies have already shown the applicability, sensitivity, accuracy and limitations of the PSICAM concept [12], even for CDOM measurements [13]-[15].

\section{MATERIALS AND METHODS}

\subsection{Field sampling}

Natural water samples were collected in the Vecht Wetlands lakes (Figure 1). The lakes are suitable for bio-optical research because the ecological conditions in the lakes are variable, ranging from oligotrophic basins to highly eutrophic lakes. Earlier analysis of the Vecht lakes area by Dekker and Peters [16] showed that the concentration range of the active optical components is large: chlorophyll- $\alpha 4-219 \mu \mathrm{g} \mathrm{L} \mathrm{L}^{-1}$, total suspended matter $1-37 \mathrm{mg} \mathrm{L}^{-1}$ and absorption by CDOM at $440 \mathrm{~nm}\left(\mathrm{~g}_{440}\right) 0.78-3.51 \mathrm{~m}^{-1}$. These ranges were reaffirmed by later research [13].

A total of 19 water samples were collected over three days at 7 stations during the summer of 2002 in the shallow eutrophic lakes Loenderveen and Terra Nova. The dates of the field days were May 6th, July 29th and September 12th. The water samples were taken just below the water surface using 1 litre Polyethylene bottles. They were temporally stored in a cooler container for transport to the laboratory, where the samples were immediately filtered.

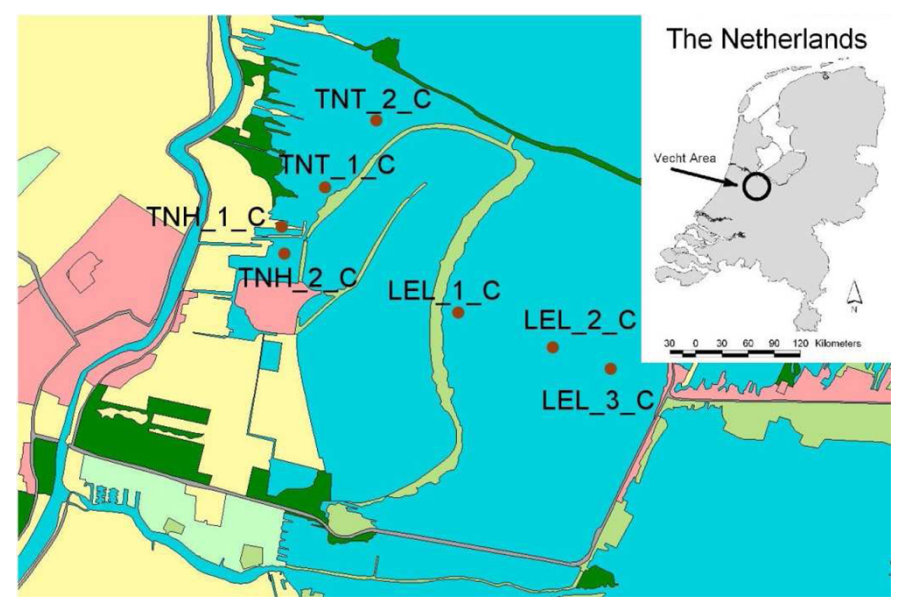

FIG. 1 Map of the Netherlands showing the Vecht Area and the lakes Loenderveen and Terra Nova and the positions of the sample stations (๔Topografische Dienst, Emmen). 


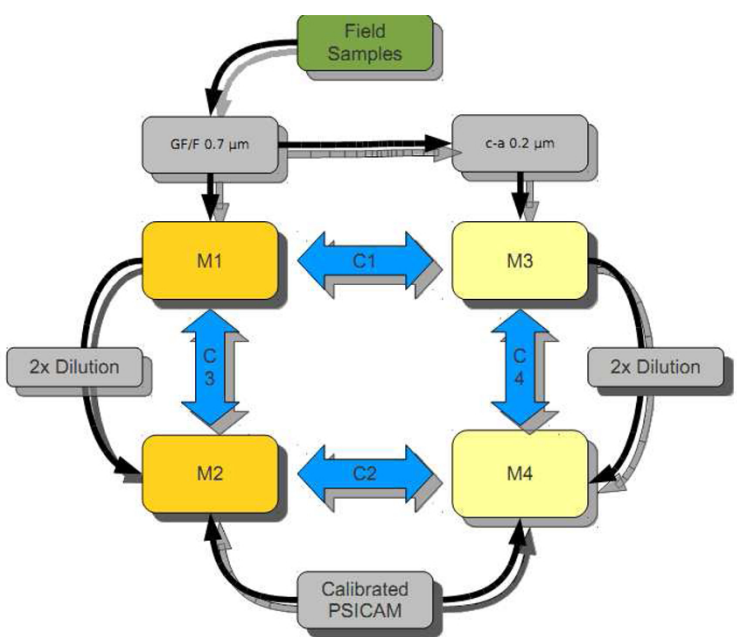

FIC. 2 Flowchart of the absorption analysis. The $M_{1}$ to $M_{4}$ are the four separate measurements techniques, while the $\mathrm{C}_{1}$ to $\mathrm{C}_{4}$ are the four inter-comparisons, shown in Figures $5,6,7$ and 8 .

\subsection{Analysis flowchart}

The analysis procedure is summarized in the flowchart, shown in Figure 2.

After collection in the field, all water samples were first filtered over Whatman GF/F filters $(0.7 \mu \mathrm{m})$. Subsequently, the filtrate of each station was again filtered over $0.2 \mu \mathrm{m}$ filters. The absorption properties of both filtrates were measured in the cuvette (M1 and M3) and with the PSICAM (M2 and M4). Based on these absorption measurements, 4 comparison experiments were performed:

- $\mathrm{C} 1$ : The cuvette $0.7 \mu \mathrm{m}$ versus cuvette $0.2 \mu \mathrm{m}$ filtered water absorption measurements. This comparison was carried out to study the differences in absorption in the cuvette for the two filter pore sizes and to characterize the overestimation of absorption measured on the $0.7 \mu \mathrm{m}$ filtrate caused by residual scattering.

- C2: The PSICAM $0.7 \mu \mathrm{m}$ versus PSICAM $0.2 \mu \mathrm{m}$ filtered water absorption measurements. This comparison was carried out to test the assumption that residual scattering has no effect on PSICAM measurements.

- C3: The PSICAM $0.7 \mu \mathrm{m}$ versus cuvette $0.7 \mu \mathrm{m}$ filtered water absorption measurements. This comparison was carried out to show the overestimation of cuvette absorption on the $0.7 \mu \mathrm{m}$ filtrate in the cuvette, and to provide a first estimate on a correction factor for historical cuvette CDOM absorption data.

- C4: The PSICAM $0.2 \mu \mathrm{m}$ versus cuvette $0.2 \mu \mathrm{m}$ filtered water absorption measurements. This comparison was carried out to test the assumption that filtering over $0.2 \mu \mathrm{m}$ takes away all scattering particles in the filtrate.

\subsection{Filtration}

The water samples were first filtered over Whatman GF/F (45 $\mathrm{mm} \varnothing, 0.7 \mu \mathrm{m}$ pore size) glass-fibre filters. A fresh filter was used for each sample. The filtrate was kept at room temperature. The absorption of the filtrate was first measured in the cuvette by an Ocean Optics Spectrophotometer. Prior to measurement in the PSICAM the filtrate was diluted two times with distilled water, because the absorption coefficients of the samples were too high to be accurately measured by the PSICAM directly. The dilutions were carried out based on weight, using a laboratory balance. For the next step the filtrate was filtered again, this time over Schleicher \& Schuell OE66 (47 $\mathrm{mm} \varnothing, 0.2 \mu \mathrm{m}$ pore size) cellulose-acetate membrane filters. Again, the absorption of the filtrate was both measured in a cuvette and, again after dilution, in the PSICAM.

\subsection{Cuvette measurements}

CDOM absorption is characterised by a strong absorption in the ultraviolet to blue wavelength region, rapidly declining towards longer wavelengths. The shape of the absorption spectrum is, based on theoretical considerations of the complex transitions in CDOM molecules, usually fitted to an exponential function [17] in order to extrapolate beyond the measured data range and to correct for measurement noise [18][20].

CDOM absorption was measured using an absorbance measurement in a $10 \mathrm{~cm}$ quartz cuvette attached to a Tungsten Halogen light source (Ocean Optics LS1-cal) at the one end and an Ocean Optics SD2000 spectrophotometer on the other. The measurement was referenced against Milli-Q water (Eqs. (4) and (5)). For this research the measured spectrum was fitted to an exponential curve, using least-squares goodness of fit in the wavelength region from 400 to $700 \mathrm{~nm}$ :

$$
a_{(\mathrm{CDOM})}(\lambda)=\Delta+a_{(\mathrm{CDOM})}\left(\lambda_{0}\right)^{*} e^{-s\left(\lambda-\lambda_{0}\right)}
$$

Here $\Delta$ is a wavelength independent offset (baseline correction), $a(C D O M)\left(\lambda_{0}\right)$ is the absorption at reference wavelength $\left(\lambda_{0}\right)$ (usually at $440 \mathrm{~nm}$, also denoted as $\mathrm{g}_{440}$ ), and $S$ is the exponential slope. Absorption coefficients given in the results paragraph are the fitted absorption coefficients at $440 \mathrm{~nm}$.

The reference wavelength of $440 \mathrm{~nm}$ was originally chosen because it corresponds approximately to the mid-point of the blue-green chlorophyll absorption maximum [4]. This chlorophyll- $\alpha$ peak is often used for chlorophyll- $\alpha$ determination of Case I waters, and by determining the CDOM absorption at the same wavelength it allows for easier comparison. Because the CDOM absorption has an exponentially declining shape without distinctive peaks, any wavelength can be used as a reference although it is preferable to use a wavelength in the blue-green region where the CDOM absorption is the highest, while remaining in the visible light region because the ultra violet domain is generally not used in water quality remote sensing due to atmospheric interference (see e.g.[7]).

\subsection{PSICAM measurements}

Since the light field inside an integrating cavity is already totally diffuse, scattering effects will (theoretically) not affect the absorption measurement in a Point Source Integrating-Cavity Absorption Meter. The operation of a PSICAM is described at length by $[13,14,21,22]$. 


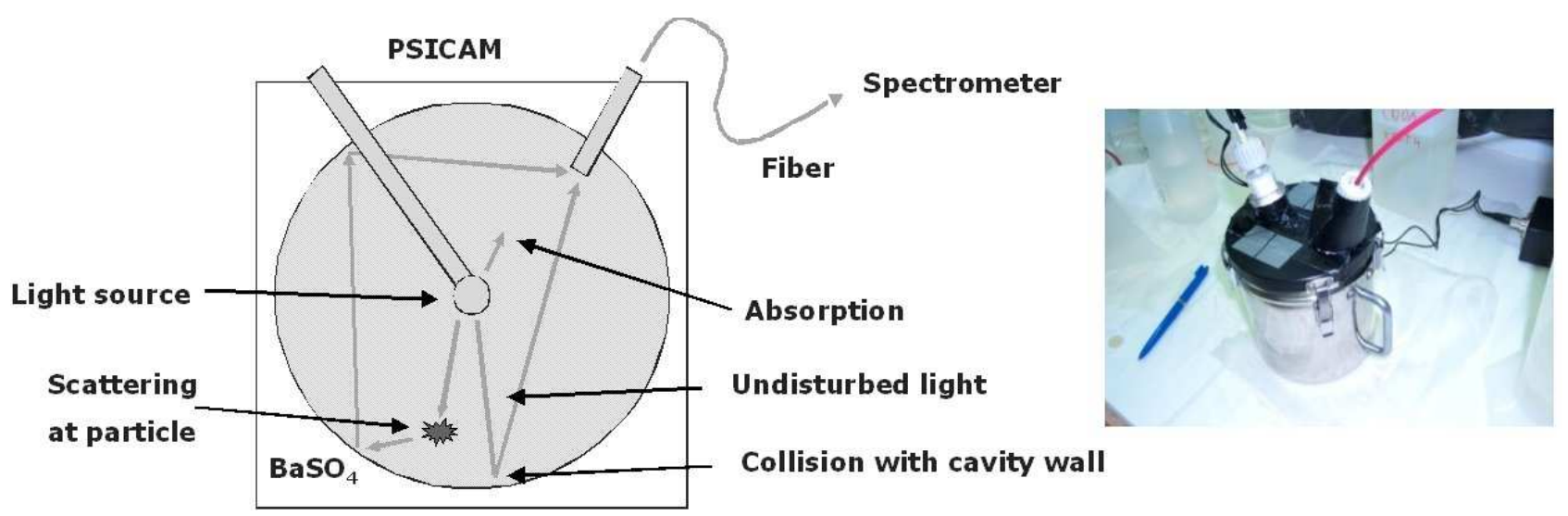

FIG. 3 Schematic overview and photograph of the CSIRO PSICAM used for this research

The PSICAM used for this research is an experimental prototype built by CSIRO (Canberra, Australia). It was designed and described by Kirk [10,11] as a spherical cavity filled with the water sample in which light is emitted by an isotropic source (a krypton-halogen light bulb customized by CSIRO) at the centre of the cavity (with a radius of $0.0505 \mathrm{~m}$ ). The emitted light is either absorbed by the medium or reflected by the highly reflecting cavity wall (filled with Barium Sulphate) or retrieved by a CC-3-UV cosine corrector leading to the Ocean Optics SD2000 spectrometer via a 400-2-UV-VIS optical fibre (see Figure 3).

The absorption of a sample inside the PSICAM is determined by Eq. (7) [11]:

$$
a=\left[\frac{\frac{4}{3} a_{(w)} r+(1-\rho)}{\frac{4}{3} r}\right] \frac{1}{\tau}-\frac{3(1-\rho)}{4 r}
$$

With $a_{(w)}$ the pure water absorption (Data from [23], completed to $800 \mathrm{~nm}$ with data from [24]) , $r$ the radius of the cavity, the cavity reflectivity $\rho$ and the transmittance $\tau$. The $\rho$ was determined by calibration of the PSICAM using a series of solutions of Aldrich Humic acid (which is similar in composition and spectral characteristics to CDOM) with known absorption coefficients [13]. As with bench spectrometers, the $\tau$ is measured against a reference of Milli-Q water and the CDOM absorption spectrum is fitted to an exponential function with an offset (Eq. (6)).

In order to obtain accurate $\mathrm{a}_{(C D O M)}$ measurements it is necessary to calibrate the PSICAM system, especially with regards to its cavity reflectivity $\rho$. It has been proposed [25] to calibrate the PSICAM by measuring the relative transmittance of two dye solutions with known absorption coefficients. The advantage of this method is that it tends to eliminate errors associated with the measurements of $\rho, \mathrm{r}$ and a [26]. Dilution series of commercially available Aldrich humic acid were used to establish a concentration-absorption relationship for the reference absorption measurements in a cuvette. Using this concentration-absorption relation, reference absorption values required for the PSICAM calibrations were calculated. Next the PSICAM calibrations were performed, resulting in a cavity reflectivity $\rho$ curve [13].

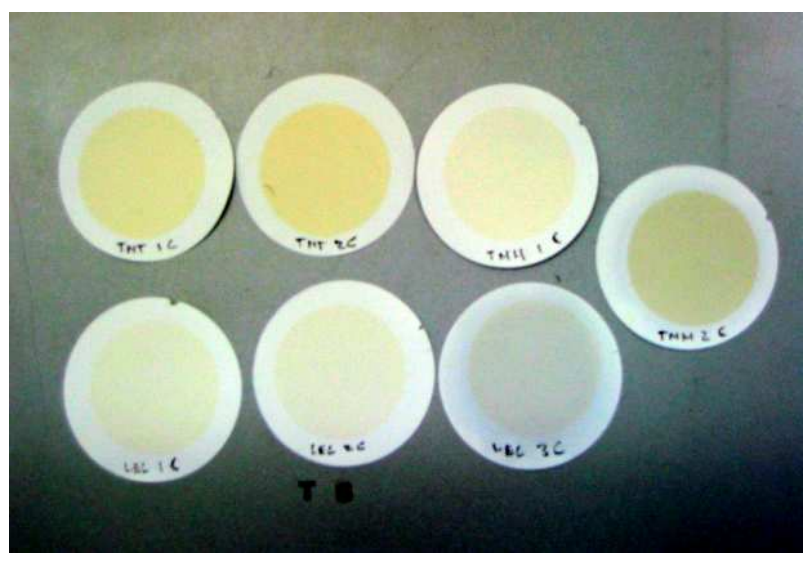

FIG. 4 Illustration of the yellowish residue that remains after second stage filtration on cellulose-acetate $0.2 \mu \mathrm{m}$ filters.

\section{RESULTS}

The yellowish residue in the $0.2-0.7 \mu \mathrm{m}$ fraction (as shown by Figure 4), that remained on some of the $0.2 \mu \mathrm{m}$ filters is indicative of the presence of an optically active group of particles in this size range. However, their mass could not be determined as the cellulose-acetate filters were not pre-weighted by very accurate mass-balances, needed to detect the small residue. Also their absorption spectrum could not be measured because the filterpad method [9] is designed for glass fibre filters and the effect of using cellulose acetate filters with a residue that contains much smaller particles is unknown.

Examples of the measured CDOM absorption spectra for this research are given in Figure 5. Please note that these spectra are already fitted using Eq. (6) and are normalised at $440 \mathrm{~nm}$ to allow easier comparison. An analysis showed that there are no significant relations between the slope and offset values of the different methods (PSICAM and cuvette) and filtration steps $(0.7$ and $0.2 \mu \mathrm{m})$. In other words, the contribution of the fraction between 0.7 and $0.2 \mu \mathrm{m}$ to the absorption spectrum has no significant spectral shape.

The results of the absorption measurements by the cuvette and the PSICAM for the two filtration steps are given in Table 1 (M1 to M4); the position of the stations can be found in Figure 1. The measured $g_{440}$ values of the PSICAM were multiplied by 2.00 to correct for the effects of dilution. For one of 


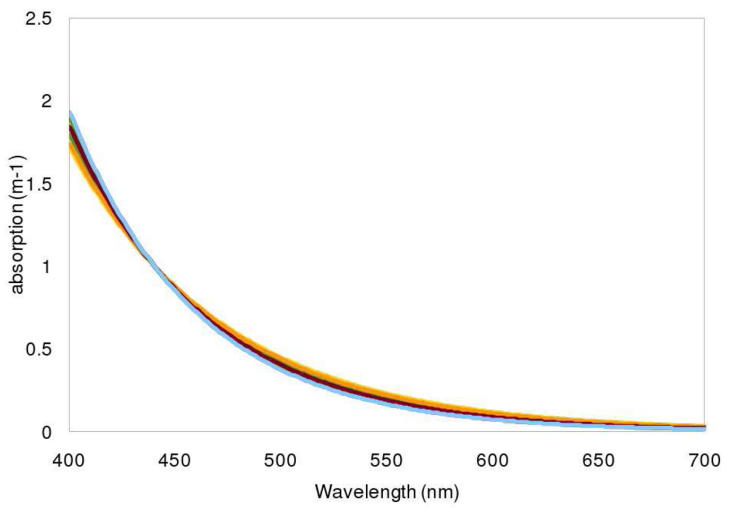

FIC. 5 Examples of the CDOM absorption spectra for the M1 data set (all normalized to $\mathrm{g}_{440}$ is 1.00 to show the spectral dependencies).

\begin{tabular}{|c|c|c|c|c|c|}
\hline Date & Station & & & & \\
\hline & & M1 & M2 & M3 & M4 \\
\hline & & $0.7 \mu \mathrm{m}$ & $0.7 \mu \mathrm{m}$ & $0.2 \mu \mathrm{m}$ & $0.2 \mu \mathrm{m}$ \\
\hline & & Cuvette & PSICAM & Cuvette & PSICAM \\
\hline \multirow{5}{*}{$\sum_{0}^{\mathrm{e}}$} & LEL-1-C & 2.55 & 2.36 & 1.98 & 2.19 \\
\hline & LEL-2-C & 2.58 & 2.27 & 2.22 & 2.14 \\
\hline & TNT-3-S & 4.27 & 3.56 & 2.88 & 2.98 \\
\hline & TNH-1-C & 3.37 & 3.08 & 2.73 & 2.77 \\
\hline & TNH-6-S & 4.51 & 4.58 & 3.92 & 4.00 \\
\hline \multirow{7}{*}{$\begin{array}{l}\bar{\Xi} \\
\stackrel{d}{d}\end{array}$} & LEL-1-C & 2.38 & 2.39 & 2.29 & 2.33 \\
\hline & LEL-2-C & 2.38 & 2.35 & 2.20 & 2.26 \\
\hline & LEL-3-C & 2.34 & 2.33 & 2.28 & 2.34 \\
\hline & TNT-1-C & 3.21 & 3.18 & 2.86 & n.a. \\
\hline & TNT-2-C & 3.18 & 3.14 & 2.83 & 3.00 \\
\hline & TNH-1-C & 4.19 & 3.83 & 3.83 & 3.67 \\
\hline & TNH-2-C & 3.86 & 3.78 & 3.45 & 3.69 \\
\hline \multirow{7}{*}{$\begin{array}{l}\stackrel{0}{0} \\
\text { ป் }\end{array}$} & LEL-1-C & 2.26 & 2.37 & 2.33 & 2.27 \\
\hline & LEL-2-C & 2.19 & 2.22 & 2.19 & 2.11 \\
\hline & LEL-3-C & 2.32 & 2.18 & 2.34 & 2.24 \\
\hline & TNT-1-C & 2.87 & 2.61 & 2.48 & 2.55 \\
\hline & TNT-2-C & 2.71 & 2.57 & 2.46 & 2.61 \\
\hline & TNH-1-C & 3.36 & 3.28 & 2.68 & 3.06 \\
\hline & TNH-2rC & 4.20 & 4.33 & 3.21 & 4.11 \\
\hline
\end{tabular}

FIG. 10 Absorption coefficients of CDOM at $440 \mathrm{~nm}\left(\mathrm{~m}^{-1}\right)$ measured in the cuvette and the PSICAM after filtration over $0.7 \mu \mathrm{m}$ and $0.2 \mu \mathrm{m}$ pore size filters.

the 19 stations (TNT-1-C on July 29th) the M4 measurement failed, leaving a set of 18 times 4 measurements.

Four comparisons were made (C1 to $\mathrm{C} 4$, Figure 2). A linear relationship was assumed between the cuvette and PSICAM absorption at $440 \mathrm{~nm}$, with the offset forced to zero: if no absorption is measured in the cuvette, then also no absorption can be measured in the PSICAM of the same sample. Paired $\mathrm{t}$-tests were performed to determine significant differences between the measured values (2-sided, $\mathrm{df}=17, \alpha 0.05$ ) and the strength of the relationships was given by the coefficient of determination of the regression, $\mathrm{R}^{2}$.

C1. From a paired t-test it followed that there is a significant difference between the cuvette 0.7 and $0.2 \mu \mathrm{m}$ filtered water sample absorption measurements (probability $\mathrm{p}=0.0003$ ). This confirms that a fraction of absorbing and scattering compounds is removed with the $0.2 \mu \mathrm{m}$ filtration. In a first approximation we assumed that the relative fraction of particles in the 0.2 to $0.7 \mu \mathrm{m}$ range is similar at all sample locations and a linear fit could be made to the data. The offset of the linear function is forced to zero because the same set-up is used. The

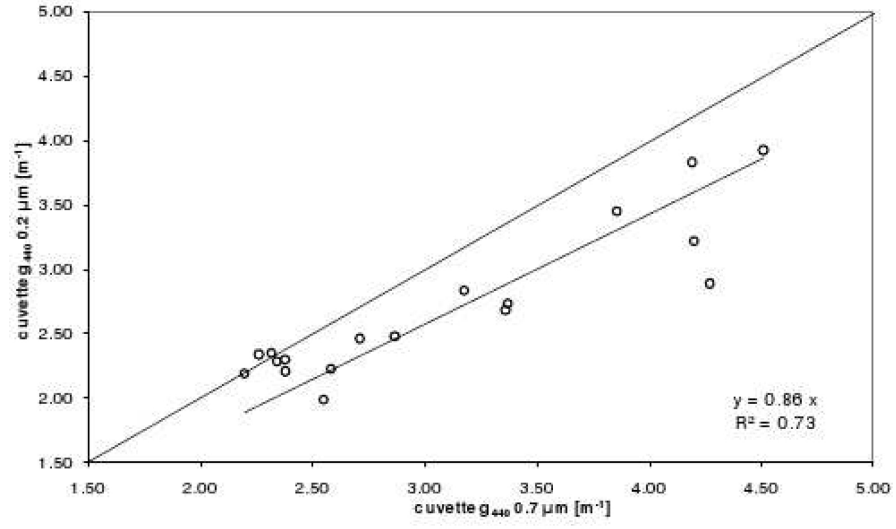

FIG. 6 The linear relation between cuvette absorption measurements at $440 \mathrm{~nm}$ after 0.7 and $0.2 \mu \mathrm{m}$ filtration.

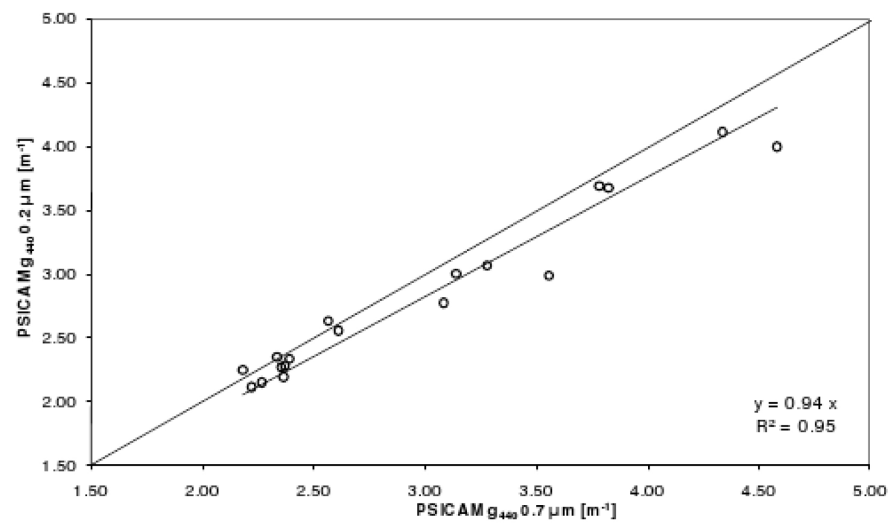

FIG. 7 The linear relation between PSICAM absorption measurements at $440 \mathrm{~nm}$ after 0.7 and $0.2 \mu \mathrm{m}$ filtration.

linear relationship between the 0.7 and $0.2 \mu \mathrm{m}$ cuvette measurements is reasonably strong $\left(R^{2}\right.$ of 0.73$)$, as is shown in Figure 6 . The average absorption loss due to the removal of matter within the size range 0.2 to $0.7 \mu \mathrm{m}$ is given by $\mathrm{a}_{(C D O M)}$ $0.2 \mu \mathrm{m}=0.86^{*} a_{(C D O M)} 0.7 \mu \mathrm{m}$ for the cuvette system, a difference of $14 \%$.

C2. There is a significant difference between the PSICAM 0.7 and $0.2 \mu \mathrm{m}$ absorption measurements according to the paired $\mathrm{t}$-test results $(\mathrm{p}=0.001)$. Analogue to the cuvette experiment, this can be explained by the removal of absorbing particles in the size range between 0.7 and $0.2 \mu \mathrm{m}$ after the $0.2 \mu \mathrm{m}$ filtration. The PSICAM measurements at 0.7 and $0.2 \mu \mathrm{m}$ have a strong linear correlation with a $\mathrm{R}^{2}$ of 0.95 as can be seen in Figure 7. The absorption loss due to the removal of matter as measured in a PSICAM is given by $\mathrm{a}_{(C D O M)} 0.2 \mu \mathrm{m}=$ $0.94^{*} a_{(C D O M)} 0.7 \mu \mathrm{m}$. When combining this relationship with the relationship derived in the previous section, the scattering contribution (b) to the attenuation measured in a cuvette (a + $\mathrm{b}=\mathrm{c}$ ) system can be quantified. The average attenuation (c) loss due to the removal of matter within the size range 0.7 and $0.2 \mu \mathrm{m}$ for the cuvette system was $14 \%$, and some $6 \%$ is the result of the actual removal of matter (following from the PSICAM results). The remaining $8 \%$ average extra attenuation in the cuvette (after $0.7 \mu \mathrm{m}$ filtration) is thus due to the scattering effects. 


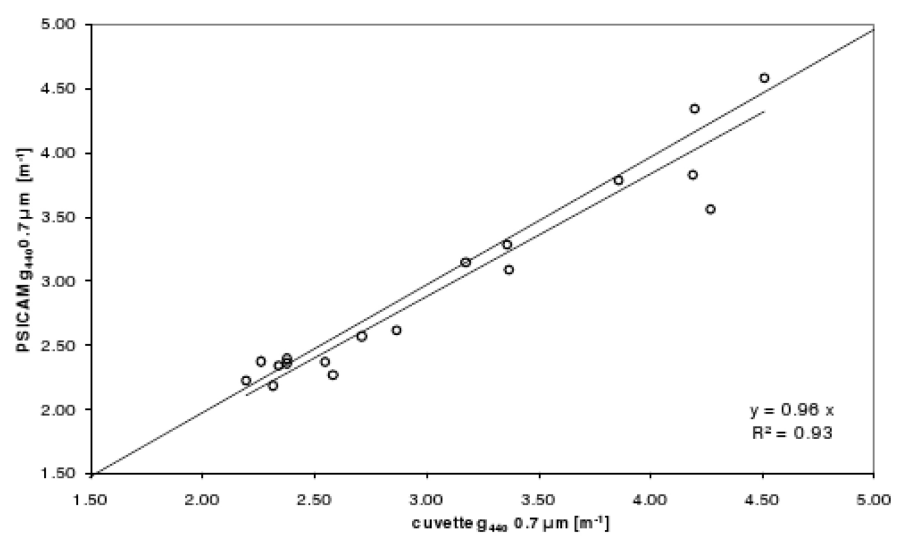

FIG. 8 The linear relation between the PSICAM and the cuvette absorption measurements after the $0.7 \mu \mathrm{m}$ filtration.

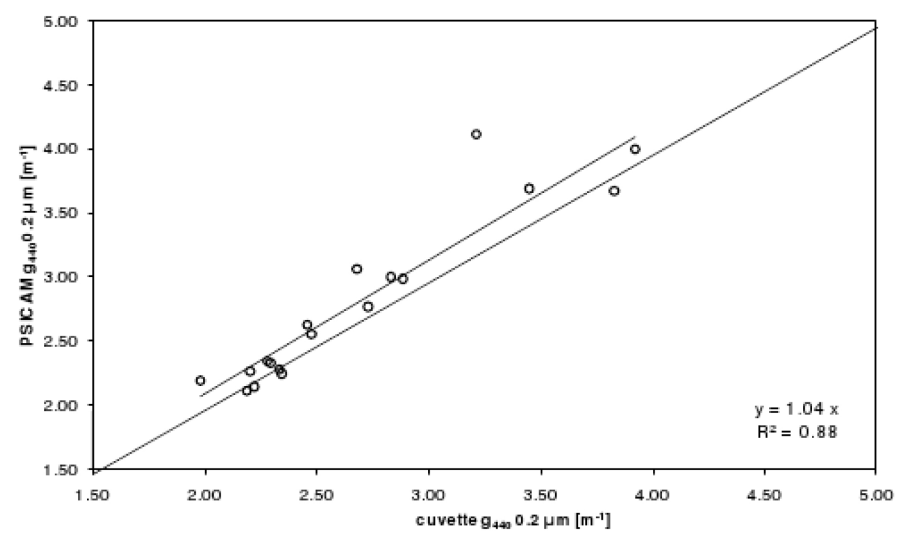

FIG. 9 The linear relation between the PSICAM and the cuvette absorption measurements after the $0.2 \mu \mathrm{m}$ filtration.

C3. There is a significant difference between the PSICAM and cuvette absorption measurements after the $0.7 \mu \mathrm{m}$ filtration ( $\mathrm{p}=0.02$ ). Figure 8 shows that the PSICAM and cuvette measurements at $0.7 \mu \mathrm{m}$ have a strong linear correlation of $\mathrm{a}(\mathrm{CDOM})$ cuvette $=0.96^{*} a_{(\mathrm{CDOM})}$ PSICAM with an $\mathrm{R}^{2}$ of 0.93 .

C4. The PSICAM and cuvette measurements at $0.2 \mu \mathrm{m}$ have a linear relationship of $\mathrm{a}_{(C D O M)}$ cuvette $=1.04^{*} a_{(C D O M)}$ PSICAM with a $\mathrm{R}^{2}$ of 0.88 (see Figure 9 ). The two data sets are not different at the $95 \%$ confidence level ( $p=0.06$ ), according to the results of the t-test. Nevertheless, the $4 \%$ difference in absorption measured after $0.2 \mu \mathrm{m}$ filtration between the PSICAM and the cuvette system is probably real and may be caused by differences in the instruments and methods themselves.

\section{DISCUSSION}

After the second filtration step all particles with sizes between 0.7 and $0.2 \mu \mathrm{m}$ are removed from the solution, which logically should result in lower absorption values. This reduction in absorption should be (relatively) the same for both the cuvette and the PSICAM, because the same fraction is removed. However, the difference between the PSICAM 0.7 and $0.2 \mu \mathrm{m}$ filtrations (C2), expressed as $0.2 \mu \mathrm{m}=0.94^{*} 0.7 \mu \mathrm{m}$, is smaller than the difference between the cuvette 0.7 and $0.2 \mu \mathrm{m}$ filtrations (C1): $0.2 \mu \mathrm{m}=0.86^{*} 0.7 \mu \mathrm{m}$.

Thus by removing the same fraction in the samples, the absorption values measured in a cuvette decrease more than those of the PSICAM. There is no relation between the absorption difference between the two filtrations and the amount of absorption present in the sample, ruling out systematic offsets between the two filtrations for the cuvette or PSICAM. The only remaining explanation therefore is that after the $0.7 \mu \mathrm{m}$ filtration the calculation of the absorption by measurement in the cuvette is affected by scattering, and therefore overestimated, whilst the absorption measurement performed in the PSICAM is not affected by scattering. The magnitude of the scattering induced difference is on average $8 \%$ (at $440 \mathrm{~nm}$ ).

The four comparisons provide a consistent description of the absorption and scattering by the sub-micron particles in the cuvette: In a direct comparison of M1 and M3 we found that the $\mathbf{C 1}$ indicates a difference of $14 \%$. If we make an indirect comparison, and relate M1 and M3 via the relations found between M1, M2, M3 and M4 we find a difference of $\mathrm{M} 3=\left(\mathbf{C} 4^{*} \mathbf{C} 2 * \mathbf{C} 3\right)^{*} \mathrm{M} 1=0.94^{*} \mathrm{M} 1$. This is identical to the absorption difference found in the PSICAM and confirms that about $8 \%$ is scattered.

There remains the fact that we found a $4 \%$ difference between the PSICAM and cuvette measurements after the $0.2 \mu \mathrm{m}$ filtration (C4). In [22] a difference of $2.4 \%$ was found for humic acid solutions in the spectral range of 400-600 nm. Next to the calibration of the inner reflection ( $\rho$ in Eq. (7)) the position and acceptance angle of the PSICAM sensor was identified as a source of inaccuracy [22]. Also for transmissometers the mean attenuation values differ markedly and in a consistent way with instrument acceptance angle [27]. Although it is expected to resolve this difference in future work, we like to stress that the basic results found above are not dependent on the discrepancy between the instruments in their measurement of the $0.2 \mu \mathrm{m}$ filtrate.

For eutrophic inland freshwaters such as found in the case study area, the size fraction between 0.7 and $0.2 \mu \mathrm{m}$ has a significant contribution to the total absorption of a sample. Thus, by applying a $0.2 \mu \mathrm{m}$ filtration step in order to measure the CDOM absorption in a cuvette without significant scattering effects, the same $0.2 \mu \mathrm{m}$ filtration step also would have to be applied for the determination of the absorption and scattering measurements of the phytoplankton and NAP. However, we realize that the $0.2 \mu \mathrm{m}$ filtration step can be very labour intensive.

In eutrophic inland waters, like the lakes presented in this article, the absorption in the blue part of the spectrum (Eq. (3)) is much larger than the backscatter term (Eq. (2)) and $R(0, \lambda)$ can be approximated by $\mathrm{R}(0, \lambda) \approx f^{*} b_{b} / a$ (see Eq. (1)). This implies that in CDOM rich waters, where the total absorption at $440 \mathrm{~nm}$ is very high $\left(2-4 \mathrm{~m}^{-1}\right.$; Table 1$)$ the errors in reflection will almost be inversely proportional to the errors in the $\mathrm{a}_{(C D O M)}$ for relatively low $\mathrm{PH}\left(<20 \mathrm{mgm}^{-3}\right)$ and NAP concentrations (see [13] for a more elaborated sensitivity analysis). Therefore the effect of an error in CDOM reference 
concentrations that are caused by residual scattering can have a significant effect on optical modelling, inhibiting accurate concentration retrieval by algorithms that are based on optical closure [28].

\section{RECOMMENDATIONS}

In this paper a case study is presented that compares PSICAM and cuvette CDOM measurements of natural inland water samples. CDOM absorption measurements made in a bench spectrometer with a cuvette are influenced by scattering effects that depend on the pore size of the filters used to collect the dissolved matter. Measurements using the PSICAM are not affected by scattering and can measure with very high precision because it has a long effective path length.

The scattering effect in the cuvette was observed and quantified. On average the $\mathrm{g}_{440}$ values of filtrate with glass-fibre filters are reduced by $14 \%$ when filtered a second time with a membrane filter. A simple visual inspection of the membrane filter residue confirmed the presence of coloured material. It was proved by PSICAM measurements that $6 \%$ of this reduction is due to reduction in absorption and $8 \%$ to scattering. Therefore, the fraction between 0.7 and $0.2 \mu \mathrm{m}$ does significantly contribute to the absorption and scattering of a sample and should not be discarded.

The PSICAM can measure true absorption differences after both 0.7 and $0.2 \mu \mathrm{m}$ filtrations. Absorption measurements with a PSICAM are comparable in complexity, speed and costs to cuvette measurements. No extra $0.2 \mu \mathrm{m}$ filtrations for the chlorophyll and suspended matter measurements would be necessary when using a PSICAM assuming that there are relatively few detritus and plankton particles smaller than $0.7 \mu \mathrm{m}$ present in the sample.

Although the results of this case study were significant, the results were derived from only 19 samples of eutrophic inland waters were available. A more comprehensive study involving a larger number of samples taken from a broader range of inland water types is recommended. Such a study might reveal a systematic overestimation of $\mathrm{a}_{(C D O M)}$ absorption (perhaps dependent on the trophic status), enabling the correction of previously determined reference CDOM absorption values.

\section{ACKN OWLEDGEMENTS}

The authors gratefully acknowledge the support provided by CSIRO Land \& Water (Australia) Environmental Earth Observation Group in hosting M. L. and providing the PSICAM. Part of the field measurements were conducted in a project carried out for the Gemeente Waterleidingen Amsterdam (Waternet). M.L was supported by the Wetlands in the Randstad programme of the Vrije Universiteit Amsterdam.

\section{References}

[1] A. Morel, and B. Gentili, "Diffuse reflectance of oceanic waters. II. Bidirectional aspects" Appl. 0pt. 32, 6864-6879 (1993).
[2] S. Sathyendranath, L. Prieur, and A. Morel, "A three-component model of ocean colour and its application to remote sensing of phytoplankton pigments in coastal waters" Int. J. Remote Sens. 10, 1373-1394 (1989).

[3] G. Tilstone, G. F. Moore, R. Sørensen, R. Doerffer, R. Pasterkamp, and P. V. Jørgensen, "REVAMP Protocols. REVAMP methodologies Regional Validation of MERIS Chlorophyll products in North Sea coastal waters" ESA Publication EVG1 - CT - 2001 âĂs 00049, Noordwijk, Netherlands (2003).

[4] J. T. 0. Kirk, Light and photosynthesis in aquatic ecosystems (2nd edition, Cambridge University Press, Cambridge, 1994).

[5] E. Aas, "Spectral Slope of Yellow Substance: Problems Caused By Small Particles" in Proceedings to Ocean Optics XV (Ocean Optics, Monac0, 2002).

[6] C. E. W. Steinberg, Ecology of Humic Substances in Freshwater, 1 (Springer, München, 2003).

[7] R. Doerffer, "Protocols for the Validation of MERIS Water Products" ESA Publication P0-TN-MEL-CS-0043, 1-42 (Noordwijk, Netherlands, 2002).

[8] S .G. H. Simis, M. Tijdens, H. L. Hoogveld, and H. J. Cons, “Optical signatures of the filamentous cyanobacterium Leptolyngbya boryana during mass viral lysis" Limnol. Oceanogr. 52, 184-197 (2007).

[9] H. G. TrÃijper, and C. M. Yentsch, “Use of glass fiber filters for the rapid preparation of in vivo absorption spectra of photosynthetic bacteria" J. Bacteriol. 94, 1255-1256 (1967).

[10] J. T. 0. Kirk, "Modeling the performance of an integrating-cavity absorption meter: theory and calculations for a spherical cavity" Appl. 0pt. 34, 4397-4408 (1995).

[11] J. T. 0. Kirk, "Point-source integrating-cavity absorption meter: theoretical principles and numerical modeling" Appl. 0pt. 36, 61236127 (1997).

[12] V. E. Brando, J. T. 0. Kirk, P. J. Daniel, K. Oubelkheir, L. Clementson, and A. G. Dekker, "Measured sensitivity of the PSICAM: effects of anisotropic light source, fluorescence, temperature and salinity" in Proceedings to Ocean Optics XVII (Ocean Optics, Fremantle, 2004).

[13] M. L. Laanen, Yellow Matters - Improving the remote sensing of Coloured Dissolved Organic Matter in inland freshwaters (Ph.D. dissertation, Vrije Universiteit Amsterdam, 2007).

[14] R. Röttgers, C. Häse, and R. Doerffer, "Determination of the particulate absorption of microalgae using a point-source integratingcavity absorption meter: verification with a photometric technique, improvements for pigment bleaching, and correction for chlorophyll fluorescence" Limnol. Oceanogr.-meth. 5, 1-12 (2007).

[15] R. Röttgers, and R. Doerffer, "Measurements of optical absorption by chromophoric dissolved organic matter using a point-source integrating cavity absorption meter" Limnol. Oceanogr.-meth. 5, 126-135 (2007).

[16] A. G. Dekker, and S. W. M. Peters, "The use of the Thematic Mapper for the analysis of eutrophic lakes: A case study in The Netherlands" Int. J. Remote Sens. 14, 799-822 (1993).

[17] K. Kalle, "The problem of the gelbstoff in the sea" Oceanogr. Mar. Biol. 4, 91-104 (1966).

[18] A. Bricaud, A. Morel, and L. Prieur, "Absorption by dissolved organic matter of the sea (yellow substance) in the UV and visible domains" Limnol. Oceanogr. 26, 43-53 (1981).

[19] S. A. Green, and N. V. Blough, “Optical absorption and fluorescence of chromophoric dissolved organic matter in natural waters" Lim- 
nol. Oceanogr. 39, 1903-1916 (1994).

[20] C. A. Stedmon, S. Markager, and H. Kaas, "Optical Properties and Signatures of Chromophoric Dissolved Organic Matter (CDOM) in Danish Coastal Waters" Estuar. Coast. Shelf S. 51, 267-278 (2000).

[21] C. J.-Y. Lerebourg, D. A. Pilgrim, G. D. Ludbrook, and R. Neal, “Development of a point source integrating cavity absorption meter" J. Opt. A-Pure Appl. 0p. 4, 56-65 (2003).

[22] R. Röttgers, W. Schoenfeld, P. Kipp, and R. Doerffer, “Practical test of a point-source integrating-cavity absorption meter (PSICAM): the performance of different collector assemblies" Appl. Opt. 44, 5549-5560 (2005).

[23] R. M. Pope, and E. S. Fry, “Absorption spectrum (380-700 nm) of pure water, II. Integrating cavity measurements" Appl. 0pt. 36, 8710-8723 (1997).

[24] H. Buiteveld, J. H. M. Hakvoort, and M. Donze, "Optical properties of pure water" in Proceedings to Ocean Optics XII 174-183 (Washington, Ocean Optics, 1994).

[25] R. A. Leathers, V. T. Downes, and C. 0. Davis, "Analysis of a pointsource integrating-cavity absorption meter" Appl. 0pt. 39, 1-10 (2000).
[26] A. G. Dekker, V. E. Brando, P. J. Daniel, J. M. Anstee, and L. Clementson, "PSICAM - FROM MYTH TO REALITY" in Proceedings to the Seventh International Conference on Remote sensing for Marine and Coastal Environments (EPA, Miami, 2002).

[27] E. Boss, W. H. Slade, M. Behrenfeld, and G. Dall’Olmo, "Acceptance angle effects on the beam attenuation in the ocean" Opt. Exp. 17, 1535-1550 (2009).

[28] H. J. Van der Woerd, and R. Pasterkamp, “HYDROPT: A fast and flexible method to retrieve chlorophyll-a from multispectral satellite observations of optically complex coastal waters" Remote Sens. Environ. 112, 1795-1807 (2008). 